\title{
Diabetes mellitus as a public health problem; a mini-review on the occasion of world diabetes day 2017 with regard to nephrology
}

\author{
Zaher Khazaei $^{1}$, Malihe Sohrabivafa ${ }^{2}$, Aboalfazl Marvi ${ }^{3^{*}}$ \\ ${ }^{1}$ Iranian Research Center on Healthy Aging, Sabzevar University of Medical Sciences, Sabzevar, Iran \\ ${ }^{2}$ Department of Health and Community Medicine, Faculty of Medicine, Dezful University of Medical Sciences, Dezful, Iran \\ ${ }^{3}$ Student Research Committee, Faculty of Health, Mazandaran University of Medical Sciences, Sari, Iran
}

\section{A R T I C L E I N F 0}

\section{Article Type:}

Mini-Review

\section{Article History:}

Received: 10 January 2018

Accepted: 10 May 2018

ePublished: 2 June 2018

\section{Keywords:}

Diabetes mellitus, Kidney failure,

Cardiovascular disease, Hypertension

Retinopathy, Nephropathy

\begin{abstract}
A B S T R A C T
Type II diabetes is predominantly preventable and managing diabetes by using standard protocols and measures such as promoting healthy living, self-care education in patients, regular screening, with the aim of early diagnosis of disease and its treatment is necessary. These modalities can potentially prevent complications and premature death due to diabetes. Therefore, according to increasing prevalence of diabetes in Iran, along with the growth of aging and lifestyle changes towards urbanization and inactivity, it is necessary to plan for the prevention, care and screening of diabetes in rural areas, especially in the cities, which still have a large proportion of unknown patients.
\end{abstract}

Implication for health policy/practice/research/medical education:

According to increasing prevalence of diabetes in Iran, along with the growth of aging and lifestyle changes towards urbanization and inactivity, it is necessary to plan for the prevention, care and screening of diabetes in rural areas, especially in the cities, which still have a large proportion of unknown patients.

Please cite this paper as: Khazaei Z, Sohrabivafa M, Marvi A. Diabetes mellitus as a public health problem; a mini-review on the occasion of world diabetes day 2017 with regard to nephrology. J Nephropharmacol. 2018;7(2):80-82.

\section{Introduction}

Diabetes is a progressive chronic disease that is characterized by an increase in blood glucose levels. The high prevalence of the disease over the past decades has caused that as a public health problem and one of the four priority diseases, non-communicable diseases considered by world leaders. The World Health Organization (WHO) reported that in 2014, around 422 million adults live with diabetes, that figure was about 108 million in 1980. The global outbreak has doubled, rising from $4.7 \%$ in 1980 to $8.5 \%$ in 2014 (1). This growth reflects an increase in risk factors associated with the disease including overweight and obesity. The outbreak in countries with lower and middle income is higher than that in high-income countries. The Eastern Mediterranean region (EMRO) has the highest ranking among $\mathrm{WHO}$ regions with a prevalence of $13.7 \%(1,2)$. The prevalence of diabetes in Iran is increasing, so that the WHO announced it at 10.3 , in 2016 . This ratio is $9.6 \%$ in men and $11.1 \%$ in women. On the other hand, the prevalence of the risk factors associated with the disease, including overweight $60.5 \%$, obesity $24.9 \%$ and low physical activity $31.9 \%$ was estimated. This ratio is considerably higher in Iranian women (3).

\section{Materials and Methods}

For this mini-review, we used a variety of sources including PubMed, Embase, Scopus and directory of open access journals (DOAJ). The search was conducted by using combinations of the following key words and/ or their equivalents; diabetes mellitus, kidney failure, cardiovascular disease, hypertension, retinopathy, 
neuropathy, nephropathy, microalbuminuria, macroalbuminuria, chronic renal failure, diabetic kidney disease, type II diabetes and end-stage renal disease.

\section{Focus on diabetic kidney disease}

Around 4 million deaths occur annually due to diabetes that included $9 \%$ of deaths (4). This figure in Iran is about $2 \%$ of all deaths (5). On the other hand, $43 \%$ of deaths due to diabetes occur before the age of 70, more importantly, the lack of control of diabetes during pregnancy can lead to adverse consequences such as maternal and infant mortality, congenital anomalies and other complications such as the birth of preterm infants $(1,6)$. Diabetes has a high direct and indirect cost to the health system annually. Most of these costs are related to the long-term complications of the disease (7). This includes the costs of preventing and treating the disease and its complications, which is done on an outpatient basis. In the study, direct costs of disease were estimated at \$ 827 billion annual (4, 7 ). When diabetes is not well managed, it causes acute and chronic complications. Acute complications of the disease include diabetic ketoacidosis in both types 1 and 2 diabetes, and hyper osmolar coma in type II diabetes. On the other hand, lowering your blood glucose level may reduce your consciousness or seizure. Late complications include cardiovascular disease, hypertension, retinopathy, neuropathy, nephropathy, cataracts and infections due to complications (8). It is the fifth leading cause of death and the leading cause of kidney failure, non-traumatic foot amputation and blindness (9). The results of the study conducted by Albright et al showed that diabetes is the main cause of blindness, renal failure, amputation and cardiovascular disease in the adult population of many countries, which is the most common cause of death (10). In another study, the prevalence of cardiovascular disease in people with diabetes was reported 2 to 4 times (11). Malik et al highlighted that among patients referring to the diabetes clinic, $77.4 \%$ had neuropathy, $33.5 \%$ had retinopathy, $18.6 \%$ had cardiovascular disease and $13.1 \%$ had nephropathy (12). Thayer et al showed that amputation in patients with diabetes is 10 times higher than those without diabetes (13).

In the study of Ford et al on patients with type II diabetes, findings revealed that $20 \%$ of patients had nephropathy, which indicated that there was a gap between the onset of the disease and the final individual for several years. Hence delays in diagnosis cause complications of diabetic notably microvascular complications (14). One of the vascular complications of diabetes is nephropathy, which is the main cause of chronic renal failure in many parts of the world (15). Nephropathy occurs in five stages, including hypertrophy of glomerular filtration and increased kidney size, glomerular damage due to increased basal remodeling thickness and mesangial matrix with normal removal of albumin, microalbuminuria (albumen excretion ranging from 300 to $30 \mathrm{mg}$ ), macro albuminuria (more proteinuria from $300 \mathrm{mg}$ ) and ultimately chronic renal failure and end-stage renal disease (ESRD) (16). Diabetes causes $40 \%$ of end-stage renal disease (ESRD), that leads to cost more than $\$ 4$ billion in the United States annually. More importantly, 30\% of people with type 1 diabetes and a lower percentage of type 2 diabetes suffer from ESRD but due to the higher incidence of type II diabetes than type I, the incidence of type II diabetes is higher (17). In some studies in diabetic patients, the outbreak of nephropathy was reported to be up to $40 \%$, and the lack of control of blood glucose in increasing micro albuminuria and reducing $\mathrm{HbA} 1 \mathrm{c}$ in less than $7 \%$ of patients was effective in reducing micro albuminuria $(10,17)$. A study in type 2 diabetes patients showed that $47 \%$ had high blood pressure, 58\% had hyperlipidemia, and the prevalence of hypertension and hyperlipidemia in patients with nephropathy was significantly higher than those without nephropathy (18). The results of a study on dialysis patients indicated that diabetes and hypertension were the main cause of renal failure, and management of care with diabetes and hypertension was effective in reducing renal insufficiency (19).

If the blood glucose, blood pressure, cholesterol, triglycerides, LDL-C, HDL-C, body mass index (BMI) are controlled in diabetic patients, and are optimal and in accordance with international standards, the complications of the disease will be prevented (20). A clinical trial study showed that with the control of diabetes and its complications, the incidence of retinopathy, nephropathy, and neuropathy decreased by $76 \%, 54 \%$ and $60 \%$, respectively. There is a significant relationship between diabetes complications and HbAlc levels; Thus, the American Diabetes Association (ADA) recommends it at least twice a year; the patient's HbAlc is checked and when this is less than 7, the patient's blood glucose monitoring is good. Along with this care, at least once a year, blood lipid levels and blood pressure measurements are essential for each patient's care (20).

\section{Conclusion}

Therefore, in order to improve the quality of diabetes care in diabetic patients, the following factors should be checked once every three months: fasting blood glucose tests, blood glucose testing 2 hours after food, HbAlc test, blood pressure measurement in both sitting and standing position and pulse rate, body mass index (BMI), examination of the lower limbs, and the care of diagnosed complications including cardiovascular disease and other related illnesses. Additionally, when there are signs of late complications during care, the patient is referred to a specialized level. On the other hand, checking blood lipids are recommended every year. Proper nutrition with the physician's opinion and providing nutritional education by a nutritionist along with education and health interventions, including lifestyle modification, advice on weight loss and increased physical activity are essential for patients every three months (18). Type II diabetes is predominantly preventable and managing diabetes by 
using standard protocols and measures such as promoting healthy living, self-care education in patients, regular screening, with the aim of early diagnosis of disease and its treatment is necessary. These modalities can potentially prevent complications and premature death due to diabetes (2). Therefore, according to increasing prevalence of diabetes in Iran, along with the growth of aging and lifestyle changes towards urbanization and inactivity, it is necessary to plan for the prevention, care and screening of diabetes in rural areas, especially in the cities, which still have a large proportion of unknown patients.

\section{Conflicts of interest}

The authors declare no conflict of interest.

\section{Ethical considerations}

Ethical issues (including plagiarism, data fabrication, double publication) have been completely observed by the authors.

\section{Authors' contribution}

All authors drafted the first version. ZKH, MS and AM edited the first draft. All authors read, reviewed and approved the final draft.

\section{Funding/Support}

None.

\section{References}

1. World Health Organization. Evaluation of Certain Food Additives and Contaminants: Eightieth Report of the Joint FAO/WHO Expert Committee on Food Additives. Geneva: World Health Organization; 2016:1-114.

2. World Health Organization. Global report on diabetes. Geneva: World Health Organization; 2016.

3. Harris SB, Tompkins JW, TeHiwi B. Call to action: A new path for improving diabetes care for Indigenous peoples, a global review. Diabetes Res Clin Pract. 2017;123:120-33.

4. Kastorini C-M, Panagiotakos DB. Mediterranean diet and diabetes prevention: Myth or fact? World J Diabetes. 2010;1:65-7. doi: 10.4239/wjd.v1.i3.65. 2010;1:65-67.

5. Nasli-Esfahani E, Farzadfar F, Kouhnavard M, GhodssiGhassemabadi R, Khajavi A, Peimani M, et al. Iran diabetes research roadmap (IDRR) study: a preliminary study on diabetes research in the world and Iran. J Diabetes Metab Disord. 2017;16:9. doi: 10.1186/s40200-017-0291-9. e.

6. Moradi G, Khazaei Z, Esmailnasab N, Roshani D, Zokaii M, Ghaderi E, et al. The relationship between maternal diseases during pregnancy and low birth weight: A nested case-control study in rural areas of Kurdistan province (West of Iran). Int J Pediatr. 2017;5:5501-14. doi: 10.22038/ IJP.2017.22666.1894

7. Farzadfar F, Murray CJ, Gakidou E, Bossert T, Namdaritabar H, Alikhani S, et al. Effectiveness of diabetes and hypertension management by rural primary healthcare workers (Behvarz workers) in Iran: a nationally representative observational study. Lancet. 2012 7;379:4754. doi: 10.1016/S0140-6736(11)61349-4.

8. Moradi S, Kerman SRJ, Rohani F, Salari F. Association between diabetes complications and leukocyte counts in Iranian patients. J Inflamm Res. 2012;5:7-11. doi: 10.2147/ JIR.S26917.

9. Sayin N, Kara N, Pekel G. Ocular complications of diabetes mellitus.World J Diabetes. 2015;6:92-108. doi: 10.4239/wjd. v6.i1.92.

10. Albright AL, Gregg EW. Preventing type 2 diabetes in communities across the US: the National Diabetes Prevention Program. Am J Prev Med. 2013; 44:S346-S51.

11. Wilmot EG, Edwardson CL, Achana FA, Davies MJ, Gorely T, Gray LJ, et al. Sedentary time in adults and the association with diabetes, cardiovascular disease and death: systematic review and meta-analysis. Diabetologia. 2012;55:2895-905. doi: 10.1007/s00125-012-2677-z.

12. Malik VS, Popkin BM, Bray GA, Després J-P, Hu FB. Sugarsweetened beverages, obesity, type 2 diabetes mellitus, and cardiovascular disease risk. Circulation. 2010;121:1356-64. doi: 10.1161/CIRCULATIONAHA.109.876185.

13. Thayer JF, Yamamoto SS, Brosschot JF. The relationship of autonomic imbalance, heart rate variability and cardiovascular disease risk factors. Int J Cardiol. 2010;141:122-31. doi: 10.1016/j.ijcard.2009.09.543

14. Ford ES, Zhao G, Li C. Pre-diabetes and the risk for cardiovascular disease: a systematic review of the evidence. J Am Coll Cardiol. 2010; 55:1310-7. doi: 10.1016/j. jacc.2009.10.060.

15. Esteghamati A, Larijani B, Aghajani MH, Ghaemi F, Kermanchi J, Shahrami A, et al. Diabetes in Iran: Prospective Analysis from First Nationwide Diabetes Report of National Program for Prevention and Control of Diabetes (NPPCD-2016). Sci Rep. 2017;7:13461. doi: 10.1038/s41598-017-13379-z.

16. Tervaert TWC, Mooyaart AL, Amann K, Cohen AH, Cook HT, Drachenberg CB, et al. Pathologic classification of diabetic nephropathy. J Am Soc Nephrol. 2010;21:556-63. doi: 10.1681/ASN.2010010010.

17. Bloom DE, Cafiero E, Jané-Llopis E, Abrahams-Gessel S, Bloom LR, Fathima S, et al. The global economic burden of noncommunicable diseases. Geneva: World Economic Forum; 2011.

18. Cushman WC, Evans GW, Byington RP, Goff Jr DC, Grimm RH Jr, Cutler JA, et al. Effects of intensive bloodpressure control in type 2 diabetes mellitus. $\mathrm{N}$ Engl J Med. 2010;362:1575-85. doi: 10.1056/NEJMoa1001286

19. Khazaei Z, Rajabfardi Z, Hatami H, Khodakarim S, Khazaei S, Zobdeh Z. Factors associated with end stage renal disease among hemodialysis patients in Tuyserkan City in 2013. Pajouhan Sci J. 2014;13(1):33-41.

20. Johnson RJ, Nakagawa T, Sanchez-Lozada LG, Shafiu M, Sundaram S, Le M, et al. Sugar, uric acid, and the etiology of diabetes and obesity. Diabetes. 2013; 62:3307-15. doi: $10.2337 / \mathrm{db} 12-1814$.

Copyright (c) 2017 The Author(s); Published by Society of Diabetic Nephropathy Prevention. This is an open-access article distributed under the terms of the Creative Commons Attribution License (http://creativecommons.org/licenses/by/4.0), which permits unrestricted use, distribution, and reproduction in any medium, provided the original work is properly cited. 